\title{
Application of the Generalized Differential Quadrature Method to the Study of Pull-In Phenomena of MEMS Switches
}

\author{
Hamed Sadeghian, Ghader Rezazadeh, and Peter M. Osterberg
}

\begin{abstract}
This paper reports on the pull-in behavior of nonlinear microelectromechanical coupled systems. The generalized differential quadrature method has been used as a high-order approximation to discretize the governing nonlinear integrodifferential equation, yielding more accurate results with a considerably smaller number of grid points. Various electrostatically actuated microstructures such as cantilever beam-type and fixed-fixed beam-type microelectromechanical systems (MEMS) switches are studied. The proposed models capture the following effects: 1) the intrinsic residual stress from fabrication processes; 2) the fringing effects of the electrical field; and 3) the nonlinear stiffening or axial stress due to beam stretching. The effects of important parameters on the mechanical performance have been studied in detail. These results are expected to be useful in the optimum design of MEMS switches or other actuators. Further, the results obtained are summarized and compared with other existing empirical and analytical models.

[2006-0101]
\end{abstract}

Index Terms-Electromechanical coupled system, generalized differential quadrature method (GDQM), microelectromechanical systems (MEMS) switch, pull-in voltage.

\section{INTRODUCTION}

$\mathbf{M}$ ICROELECTROMECHANICAL systems (MEMS) devices are rapidly gaining popularity in a variety of industrial applications such as aerospace, automotive, and biomedical industries. MEMS devices are generally classified according to their actuation mechanisms. Actuation mechanisms for MEMS vary depending on the suitability to the application at hand. The most common actuation mechanisms are electrostatic, pneumatic, thermal, and piezoelectric [1]. Electrostatically actuated devices form a broad class of MEMS devices due to their simplicity, as they require few mechanical components and small voltage levels for actuation [1]. The structural elements that are used in MEMS devices are typically simple elements including beams (or microbeams), plates, and membranes. Electrostatically actuated microbeams (e.g., can-

Manuscript received May 28, 2006; revised July 19, 2007. Subject Editor K. Naiafi.

H. Sadeghian is with the Structural Optimization and Computational Mechanics Group, Precision and Microsystems Engineering Department, and the Electronic Instrumentation Laboratory, Microelectronics Department, Delft University of Technology, 2628 CD Delft, The Netherlands (e-mail: h.SadeghianMarnani@tudelft.nl).

G. Rezazadeh is with the Mechanical Engineering Department, Faculty of Engineering, Urmia University, 57135 Urmia, Iran (e-mail: g.rezazadeh@ mail.urmia.ac.ir).

P. M. Osterberg is with the Electrical Engineering Department, University of Portland, Portland, OR 97203-5743 USA (e-mail: oster@up.edu).

Digital Object Identifier 10.1109/JMEMS.2007.909237 tilever and fixed-fixed microbeams) are used in many MEMS devices such as capacitive MEMS switches and resonant sensors. Manufacturing and design of these devices are, to some extent, in a more mature stage than some other MEMS devices.

MEMS microbeams are susceptible to an instability that is known as pull-in. When the applied voltage is increased beyond a critical value, which is called the pull-in voltage, stable equilibrium positions of the microbeam cease to exist. Pull-in instability [2] greatly limits the stable range of operation of microbeams. In most cases, it would be highly desirable to delay the onset of pull-in for better performance of the device. The pull-in voltage depends on the interaction of the electrostatic forces (generated by the applied voltage) and the structural stiffness of the microbeam. Previous work addressing pull-in instability has taken both electrical and mechanical approaches. In 1994, Osterberg et al. [3] proposed three pull-in models to elucidate the pull-in behavior of cantilever, fixed-fixed, and circulate plate microstructures including a 1-D lumped parallel plate spring model, a 2-D finite-difference model, and a 3-D analytical model. These were compared to a 3-D numerical computer model using the CoSolve-EM simulator [4]. In 1997, Legtenberg et al. [5] proposed the use of curved electrodes to determine the characteristics of large-displacement actuators. A 3-D coupled electromechanical model was presented to understand the interaction between the deflection of the actuator and the electrostatic force where the Rayleigh-Ritz method was applied to solve the 1-D small deflection energy model. Hung and Senturia [6], [7] presented a technique where the electrostatic force is applied only to a certain portion of a beam. This design uses lever action to achieve full gap deflection, while keeping the electrostatic gap deflection within the one-third range. Sadeghian and Rezazadeh [8], [9] performed a formal numerical investigation of the behavior of MEMS switches for the following purposes: 1) to capture important second-order effects including residual stress; 2) to develop a new method to measure the stress gradient in cantilever beam-type switches; and 3) to analyze the effect of real anchors of fixed-fixed beams on pull-in phenomena. Sadeghian et al. [10] presented a technique where the electrostatic force is applied only to a certain portion of a beam to decrease the pull-in voltage. In the case of multilayer microstructures, Rezazadeh et al. [11], [12] developed a comprehensive model to study the pullin behavior of nonlinear electromechanical coupled systems where the effects of both thickness and Young's modulus of each layer on the pull-in voltage were captured. 


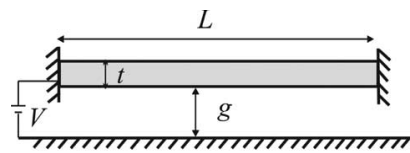

(a)

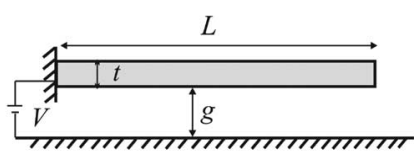

(b)
Fig. 1. Schematic diagrams of (a) fixed-fixed beam-type MEMS switch and (b) cantilever beam-type MEMS switch.

In this paper, the pull-in behaviors of MEMS switches with various electrostatically actuated microstructures such as cantilever beam-type and fixed-fixed beam-type are studied. The influence of various parameters on the pull-in voltage, such as nonlinear stiffening, fringing fields [13], trapezoidal cross section [14], residual stress [15], and axial stress, has been studied. The static pull-in voltage is determined by coupling the mechanics to a nonlinear voltage-dependent electrostatic pressure term and finding the lowest voltage at which the system is unstable. The method for solving the governing integrodifferential equation in this paper is the generalized differential quadrature method (GDQM). The predecessor to GDQM was the differential quadrature method (DQM). It was originally introduced by Bellman et al. [16] as a simple and highly efficient numerical technique. One essential issue pertaining to the method is how to efficiently and accurately determine its weighting coefficients. The earlier approach (i.e., DQM), which requires solving algebraic equations with an ill-conditioned Vandermonde matrix, is neither efficient nor accurate when the number of grid points is large [16], [17]. Based on the Lagrange interpolation, Shu and Richards [18] presented GDQM where the determination of weighting coefficients of the differential quadrature discretization is generalized under the analysis of a high-order polynomial approximation and the analysis of a linear vector space that make GDQM more accurate and efficient.

\section{MODEL DESCRIPTION}

MEMS switches are generally modeled as a cantilever beam structure or a fixed-fixed beam structure (see Fig. 1). The device consists of a thin film metal membrane, which is called the "beam," suspended over a dielectric film deposited on top of the center conductor and fixed at one side in the case of the cantilever and fixed at both ends to the ground conductors in the case of the fixed-fixed beam. When a voltage is applied between the beam and substrate, the attractive electrostatic pressure pulls the beam down toward the dielectric film. The dielectric film serves to prevent stiction between the beam and substrate and provides a low impedance path between the two contacts. The switch can be fabricated using surface micromachining techniques, electroplating techniques, and dry releasing techniques that are compatible with millimeter-wave integrated circuit fabrication processes. A brief fabrication sequence for the fixed-fixed beam MEMS switches that is shown in Fig. 1(a) is described in [19].

The choice of the appropriate structural analysis model of the microbeam depends on the magnitude of the deflection compared to the thickness of the microbeam. Since the deformation of the microbeam will be on the same order of magnitude as the initial gap dimension $g$, the proper form of the structural model will depend on the ratio $g / t$, where $t$ is the thickness of the beam. For values of $g / t \leq 1$, a linear Euler-Bernoulli model will be an adequate representation of the microbeam behavior. In the case of fixed-fixed beams, for moderate values of $g / t$ (i.e., $g / t \approx 1$ ), bending-stretching coupling terms need to be taken into account, and for large values of $g / t$ (i.e., $g / t \geq 2$ ), or when the microbeam is initially under large tension, the fixed-fixed microbeam should be modeled as a membrane with geometrical nonlinearity.

\section{Nonlinear Distributed ElectromechaniCAL COUPLED MODEL}

When a driving voltage is applied between the electrodes, the electrostatic pressure deflects the beam. The mechanical bending strain energy $U_{m}$ of the beam and the electrical coenergy $U_{e}^{*}$ (which is the sum of the electrostatic energy stored between the upper and lower electrodes of the beam and the electrostatic energy of the voltage source) are given by

$$
\begin{aligned}
U_{m} & =\int_{0}^{L} \int_{A} \frac{\tilde{E}}{2}\left(-z \frac{d^{2} u}{d x^{2}}\right)^{2} d A d x=\int_{0}^{L} \frac{\tilde{E} I}{2}\left(\frac{d^{2} u}{d x^{2}}\right)^{2} d x \\
U_{e}^{*} & =\frac{1}{2} \int_{0}^{L} \frac{\varepsilon_{0} w V^{2}}{(g-u(x))} d x
\end{aligned}
$$

where $z$ is the coordinate in the load direction with the origin in the centroid of the cross section, $\varepsilon_{0}$ is the permittivity of the vacuum, $w$ is the width of the beam, $g$ is the initial gap between the upper and lower electrodes, $u(x)$ is the deflection, and $V$ is the applied voltage between the movable upper plate and the fixed ground plate on the substrate. $\tilde{E}$ is dependent on the beam width $w$ and film thickness $t$ [20]. A beam is considered wide when $w \geq 5 t$. Wide beams exhibit plane-strain conditions, and therefore, $\tilde{E}$ becomes the plate modulus, i.e., $E /\left(1-\nu^{2}\right)$, where $E$ is the Young's modulus. A beam is considered narrow when $w<5 t$. In this case, $\tilde{E}$ simply becomes the Young's modulus $E$. The quantity $I$ (the moment of inertia of the cross section) is equal to $\left(w t^{3}\right) / 12$.

The energy stored in the beam due to residual and axial forces is

$$
U_{s}=\int_{0}^{L}\left(T_{r}+T_{a}\right)\left(\frac{d u}{d x}\right)^{2} d x
$$

where $T_{r}$ and $T_{a}$ are the residual and axial forces, respectively.

The total potential energy $U$ of the system is

$$
U=U_{m}-U_{s}+U_{e}
$$

The variation of total energy is zero at the equilibrium position, i.e.,

$$
\delta U=\delta U_{m}-\delta U_{s}+\delta U_{e}=\delta U_{m}-\delta U_{s}-\delta U_{e}^{*}=0 .
$$


Now by considering the function $U(u)$ as follows:

$$
U(u)=\int_{0}^{L} F\left(x, u, u_{x}, u_{x x}\right) d x
$$

we can derive the nonlinear differential equation of the system. The necessary conditions for $u(x)$ to be a minimizing function can be derived if we integrate the second and third terms by parts. The result is given as follows:

$$
\begin{aligned}
\delta U= & \int_{0}^{L}\left[\frac{\partial F}{\partial u}-\frac{d}{d x}\left(\frac{\partial F}{\partial u_{x}}\right)+\frac{d^{2}}{d x^{2}}\left(\frac{\partial F}{\partial u_{x x}}\right)\right] \delta u d x \\
& +\left.\left[\frac{\partial F}{\partial u_{x}}-\frac{d}{d x}\left(\frac{\partial F}{\partial u_{x x}}\right)\right] \delta u\right|_{0} ^{L}+\left[\left(\frac{\partial F}{\partial u_{x x}}\right) \delta u+x\right]_{0}^{L} \\
= & 0 .
\end{aligned}
$$

The first part of (7) is called the Euler-Lagrange equation and is the differential equation that $u(x)$ must satisfy. The second and third parts are satisfied by the variations of $u$ and $u_{x}$, which are zero on the boundaries. Substituting our equation for total potential energy in (7) yields

$$
\begin{aligned}
\delta U=\int_{0}^{L}\left(\frac{d^{2}}{d x^{2}}\left(E I \frac{d^{2} u}{d x^{2}}\right)\right. & -\frac{d}{d x}\left(\left(T_{r}+T_{a}\right) \frac{d u}{d x}\right) \\
& \left.-\frac{\varepsilon_{0} w V^{2}}{2(g-u(x))^{2}}\right) \delta u d x=0
\end{aligned}
$$

where $\delta u$ is an arbitrary function. Therefore, we obtain the following expression:

$$
\frac{d^{2}}{d x^{2}}\left(\tilde{E} I \frac{d^{2} u}{d x^{2}}\right)-\left(T_{r}+T_{a}\right) \frac{d^{2} u}{d x^{2}}=\frac{\varepsilon_{0} w V^{2}}{2(g-u(x))^{2}} .
$$

\section{A. Residual Stress}

Residual stress, due to the inconsistency of both the thermal expansion coefficient and the crystal lattice period between the substrate and thin film, is unavoidable in surface micromachining techniques. Accurate and reliable data for residual stress are crucial to the proper design of MEMS devices that are related to these techniques [21], [22]. Therefore, residual stress is an attractive research topic in microsystems technology. Considering the fabrication sequence of radio frequency MEMS switches, residual force can be expressed as

$$
T_{r}=\sigma_{r}(1-\nu) w t
$$

where $\sigma_{r}$ is the biaxial residual stress [23] (equal to zero for cantilever beams), and $\nu$ is the Poisson's ratio.

\section{B. Axial Stress}

When a beam is in tension, the actual beam length $L^{\prime}$ is longer than the original length $L$. Although there is no displacement in the $x$-direction at the beam ends, the bending of the beam generates an axial force, i.e.,

$$
T_{a}=\frac{\tilde{E} A}{L}\left(L^{\prime}-L\right) \approx \frac{\tilde{E} w t}{2 L} \int_{0}^{L}\left(\frac{d u}{d x}\right)^{2} d x
$$

where $L^{\prime}$ is the actual length along the centerline of the beam. This length is calculated by integrating the arc length $d s$ along the curved beam based on the cubic shape functions for small angle beam deflection.

\section{Fringing Effect of Electrical Field}

A uniform electric field cannot abruptly drop to zero at an edge. In an actual situation, there always exists a "fringing field." Therefore, a more realistic model including the "fringing field" is necessary. The first-order fringing field correction [15], [24] is expressed as

$$
f_{f}=0.65\left[\frac{g-u(x)}{w}\right] .
$$

The driving electrostatic pressure $q$, which is the term on the right-hand side of (9), becomes

$$
q(u, V)=\frac{\varepsilon_{0} V^{2} w}{2[g-u(x)]^{2}}\left(1+f_{f}\right) .
$$

\section{Stress Gradients}

Nonuniform stresses in the film thickness create built-in moments, which in released cantilevers cause them to curl out of plane. Because of the large possible deflections in gap from these structures, the electrostatic pressure is significantly modified from the corresponding uniformly flat case and the pull-in voltages can be significantly shifted. Because the stress gradient is assumed to be uniform in-plane, the cantilever experiences a constant bending moment along its length when it is flat and has a constant radius of curvature $R_{c}$ after it is released. $f_{c}$ is the modification term for stress gradient [23] and is expressed as

$$
f_{c} \approx 1+0.5096\left(\frac{L^{2}}{g R_{c}}\right)+0.0006347\left(\frac{L^{2}}{g R_{c}}\right)^{2} .
$$

Cantilevers curl out of plane due to stress gradients through the polysilicon film thickness. An optical tip deflection measurement was used to determine the curling radius $R_{c}$ of $40 \pm 0.3 \mathrm{~mm}$ from the 500-mm-long cantilever [23]. The modification term for stress gradient is calculated using the results in [23]. This modification term is then multiplied by the pullin voltages from the ideal uniformly flat case to yield the real 

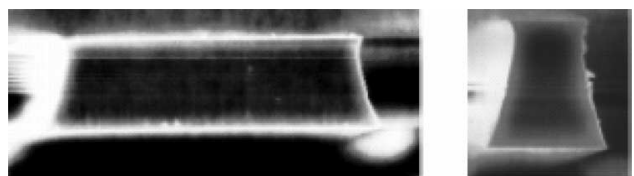

Fig. 2. SEM cross sections of cantilevers [23].

pull-in voltage of cantilevers with initial deformation due to stress gradients.

\section{E. Trapezoidal Cross Section}

The SEM images in Fig. 2 are used to obtain the trapezoidal cross section due to plasma etching process [23]. The compliance of the beam's trapezoidal cross section on the bottom face of the beam is included in the effective moment of inertia of the cross section, i.e.,

$$
I=\left(t^{3} / 36\right)\left\{\left(w^{2}+4 w w^{\prime}+w^{\prime 2}\right) /\left(w+w^{\prime}\right)\right\}
$$

where $w^{\prime}$ is the bottom width of the beam, and $w$ is the top width of the beam.

\section{GQDM}

GDQM is used to transform the aforementioned nonlinear integro-differential equations into the corresponding discrete forms and is an extension of the predecessor method, i.e., DQM. The fundamental idea behind DQM is that the differentorder partial derivatives of a function at a given point can be approximated by a weighted sum of function values at all discrete points in the variable domain. Therefore, an $m$ thorder derivative of a function $u(x)$ at a given point $x_{i}$ can be determined. GDQM uses the same basic concept as DQM. Specifically, the derivative of a sufficiently smooth function with respect to a coordinate direction at a discrete point can be approximated by a weighted linear sum of functional values at all discrete points in that direction.

It has been observed that GDQM has two advantages over DQM as follows: 1) there is no restriction required on the distribution and number of discrete grid points and 2) the weighting coefficient $w_{i j}^{(m)}$ can be determined using a simple recurrence relation instead of solving a set of linear algebraic equations. By applying GDQM with $N$ discrete points [25], we obtain the following expressions:

$$
\begin{aligned}
\left.\frac{\partial^{m} u(x)}{\partial x^{m}}\right|_{x=x_{i}}= & \sum_{j=1}^{N} w_{i j}^{(m)} u_{j}, \quad i=1,2, \ldots, N \\
w_{i j}^{(1)}= & \frac{1}{x_{j}-x_{i}} \prod_{\substack{k \neq i \\
k \neq j \\
k=1}}^{N} \frac{x_{i}-x_{k}}{x_{j}-x_{k}}, \\
& i=1,2, \ldots, N ; \quad j=1,2, \ldots, N \\
w_{i i}^{(1)}= & \sum_{k \neq i}^{N} \frac{1}{x_{i}-x_{k}}, \quad i=1,2, \ldots N .
\end{aligned}
$$

The weighting coefficients of higher order derivatives can also be determined by matrix multiplication as follows [21]:

$$
\begin{aligned}
& w_{i j}^{(2)}=\sum_{k=1}^{N} w_{i k}^{(1)} w_{k j}^{(1)} \\
& w_{i j}^{(3)}=\sum_{k=1}^{N} w_{i k}^{(1)} w_{k j}^{(2)} \\
& w_{i j}^{(4)}=\sum_{k=1}^{N} w_{i k}^{(1)} w_{k j}^{(3)} .
\end{aligned}
$$

For a cantilever beam, the corresponding boundary conditions are

$$
u(0)=0,\left.\quad \frac{d u}{d x}\right|_{x=0}=0,\left.\quad \frac{d^{2} u}{d x^{2}}\right|_{x=l}=0,\left.\quad \frac{d^{3} u}{d x^{3}}\right|_{x=l}=0
$$

The aforementioned set of boundary conditions yields the following weighting coefficient matrices:

$w_{i j}^{(1)}=\left[\begin{array}{ccccc}0 & 0 & \cdots & 0 & 0 \\ 0 & w_{22}^{(1)} & \cdots & w_{2, N-1}^{(1)} & w_{2, N}^{(1)} \\ \vdots & \vdots & \ddots & \vdots & \vdots \\ 0 & w_{N-1,2}^{(1)} & \cdots & w_{N-1, N-1}^{(1)} & w_{N-1, N}^{(1)} \\ 0 & w_{N, 2}^{(1)} & \cdots & w_{N, N-1}^{(1)} & w_{N, N}^{(1)}\end{array}\right]$

$w_{i j}^{(2)}=\left[\begin{array}{ccccc}w_{11}^{(1)} & w_{12}^{(1)} & \cdots & w_{1, N-1}^{(1)} & w_{1, N}^{(1)} \\ w_{21}^{(1)} & w_{22}^{(1)} & \cdots & w_{2, N-1}^{(1)} & w_{2, N}^{(1)} \\ \vdots & \vdots & \ddots & \vdots & \vdots \\ w_{N-1,1}^{(1)} & w_{N-1,2}^{(1)} & \cdots & w_{N-1, N-1}^{(1)} & w_{N-1, N}^{(1)} \\ w_{N, 1}^{(1)} & w_{N, 2}^{(1)} & \cdots & w_{N, N-1}^{(1)} & w_{N, N}^{(1)}\end{array}\right]\left[w_{i j}^{(1)}\right]$

$w_{i j}^{(3)}=\left[\begin{array}{ccccc}w_{11}^{(1)} & w_{12}^{(1)} & \cdots & w_{1, N-1}^{(1)} & w_{1, N}^{(1)} \\ w_{21}^{(1)} & w_{22}^{(1)} & \cdots & w_{2, N-1}^{(1)} & w_{2, N}^{(1)} \\ \vdots & \vdots & \ddots & \vdots & \vdots \\ w_{N-1,1}^{(1)} & w_{N-1,2}^{(1)} & \cdots & w_{N-1, N-1}^{(1)} & w_{N-1, N}^{(1)} \\ 0 & 0 & \cdots & 0 & 0\end{array}\right]\left[w_{i j}^{(2)}\right]$

$$
w_{i j}^{(4)}=\left[\begin{array}{ccccc}
w_{11}^{(1)} & w_{12}^{(1)} & \cdots & w_{1, N-1}^{(1)} & w_{1, N}^{(1)} \\
w_{21}^{(1)} & w_{22}^{(1)} & \cdots & w_{2, N-1}^{(1)} & w_{2, N}^{(1)} \\
\vdots & \vdots & \ddots & \vdots & \vdots \\
w_{N-1,1}^{(1)} & w_{N-1,2}^{(1)} & \cdots & w_{N-1, N-1}^{(1)} & w_{N-1, N}^{(1)} \\
w_{N, 1}^{(1)} & w_{N, 2}^{(1)} & \cdots & w_{N, N-1}^{(1)} & w_{N, N}^{(1)}
\end{array}\right]\left[w_{i j}^{(3)}\right] .
$$

For a fixed-fixed beam, the matrices of weighting coefficients using GDQM and considering the boundary 
conditions are

$$
\begin{aligned}
& w_{i j}^{(1)}=\left[\begin{array}{ccccc}
0 & 0 & \cdots & 0 & 0 \\
0 & w_{22}^{(1)} & \cdots & w_{2, N-1}^{(1)} & 0 \\
\vdots & \vdots & \ddots & \vdots & \vdots \\
0 & w_{N-1,2}^{(1)} & \cdots & w_{N-1, N-1}^{(1)} & 0 \\
0 & 0 & \cdots & 0 & 0
\end{array}\right] \\
& w_{i j}^{(3)}=\left[\begin{array}{ccccc}
w_{11}^{(1)} & w_{12}^{(1)} & \cdots & w_{1, N-1}^{(1)} & w_{1, N}^{(1)} \\
w_{21}^{(1)} & w_{22}^{(1)} & \cdots & w_{2, N-1}^{(1)} & w_{2, N}^{(1)} \\
\vdots & \vdots & \ddots & \vdots & \vdots \\
w_{N-1,1}^{(1)} & w_{N-1,2}^{(1)} & \cdots & w_{N-1, N-1}^{(1)} & w_{N-1, N}^{(1)} \\
w_{N, 1}^{(1)} & w_{N, 2}^{(1)} & \cdots & w_{N, N-1}^{(1)} & w_{N, N}^{(1)}
\end{array}\right]\left[w_{i j}^{(2)}\right]
\end{aligned}
$$

$$
w_{i j}^{(4)}=\left[\begin{array}{ccccc}
w_{11}^{(1)} & w_{12}^{(1)} & \cdots & w_{1, N-1}^{(1)} & w_{1, N}^{(1)} \\
w_{21}^{(1)} & w_{22}^{(1)} & \cdots & w_{2, N-1}^{(1)} & w_{2, N}^{(1)} \\
\vdots & \vdots & \ddots & \vdots & \vdots \\
w_{N-1,1}^{(1)} & w_{N-1,2}^{(1)} & \cdots & w_{N-1, N-1}^{(1)} & w_{N-1, N}^{(1)} \\
w_{N, 1}^{(1)} & w_{N, 2}^{(1)} & \cdots & w_{N, N-1}^{(1)} & w_{N, N}^{(1)}
\end{array}\right]\left[w_{i j}^{(3)}\right] .
$$

The Newton-Raphson method is a standard procedure for solving a set of nonlinear algebraic equations that result from the application of GDQM to nonlinear differential and integrodifferential equations.

\section{RESUlts}

The pull-in results for a cantilever beam made of singlecrystalline silicon subjected to a voltage are now presented. Specifically, the cantilever beam's pull-in results predicted from the proposed GDQM algorithm are compared with the results predicted in [27]. These pull-in results are also compared with the experimental results [27]. The cantilever beam's geometric and material properties are given as follows [27]: Young's modulus $E$ is $155.8 \mathrm{GPa}$, Poisson's ratio is 0.06 , length of the beam $L$ is $20 \mathrm{~mm}$, width of the beam $w$ is $5 \mathrm{~mm}$, thickness of the beam $t$ is $57 \mu \mathrm{m}$, initial gap $g$ is $92 \mu \mathrm{m}$, and the permittivity of air is $8.85 \mathrm{pF} / \mathrm{m}$. The stress gradient is estimated to be $0.2 \mathrm{MPa} / \mu \mathrm{m}$. This value is used to check the influence on the pull-in voltage. Table I shows the end gap versus voltage simulation results (GDQM and [27]), which are compared with the experimental results. $\Delta \%$ in Table I is the difference between the results from
TABLE I

END GaP Versus Voltage for CANTILEVER BEAM

\begin{tabular}{|c|c|c|c|c|}
\hline Voltage & $\begin{array}{c}\text { End Gap }(\mu \mathrm{m}) \\
(\text { GDQM) }\end{array}$ & $\begin{array}{c}\text { End Gap }(\mu \mathrm{m}) \\
{[27]}\end{array}$ & $\begin{array}{c}\text { Eng Gap }(\mu \mathrm{m}) \\
\text { Experimental [27] }\end{array}$ & $\begin{array}{c}\Delta \% \\
(\text { GDQM vs. } \\
\text { Experimental })\end{array}$ \\
\hline 20 & 90.2 & 90.2 & 90.5 & 0.3 \\
\hline 40 & 84.1 & 84.3 & 84.6 & 0.6 \\
\hline 60 & 69.1 & 71.5 & 70.0 & 1.2 \\
\hline 65 & 59.6 & 67.2 & 64.0 & 6.8 \\
\hline
\end{tabular}

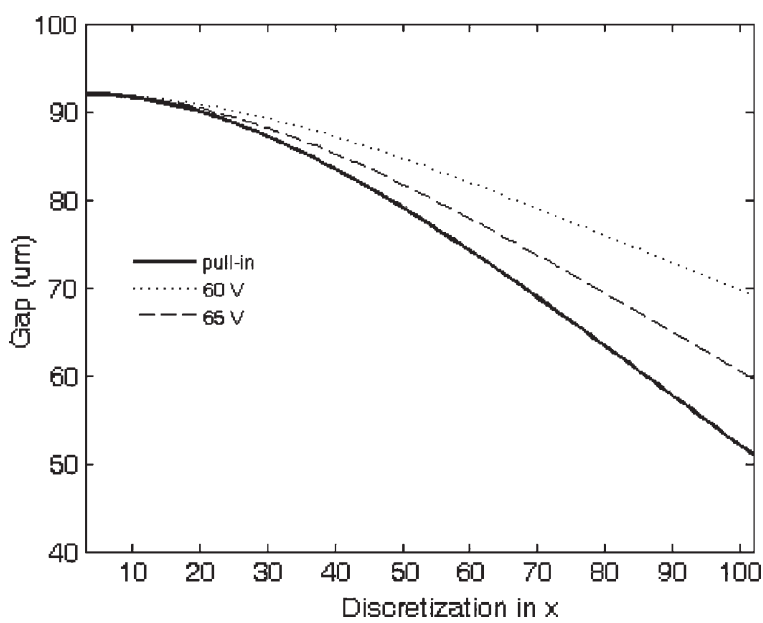

Fig. 3. GDQM-modeled deflection of cantilever beam at different voltages generated by MATLAB 7.

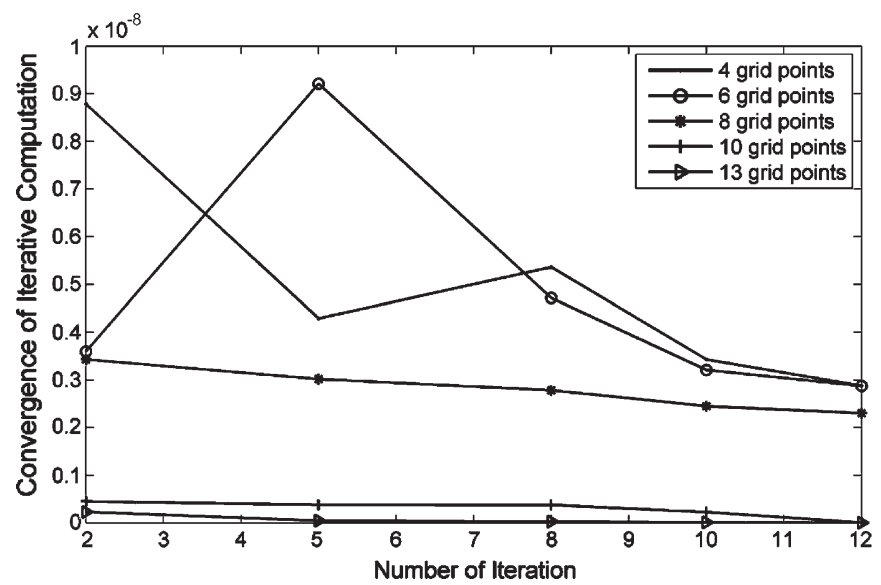

Fig. 4. Rate of convergence with respect to the number of grid points evaluated with GDQM.

the proposed model and the experimental data. Fig. 3 shows the deflection of the cantilever beam at pull-in and two voltages just below pull-in from Table I using GDQM and MATLAB 7. In the proposed GDQM, only 13 grid points on the beam are used, and a convergence with a tolerance of $10^{-12}$ was achieved in less than ten iterations. The rate of convergence with respect to the number of grid points has been demonstrated in Fig. 4. The estimated pull-in voltage is approximately 66.4 using GDQM compared to 68.5 in [27].

For fixed-fixed beams, the results predicted by employing the 2-D distributed model and 3-D micro-electro-mechanical computer-aided-design (MEMCAD) simulation [28] are compared with those obtained using the proposed GDQM algorithm to demonstrate the feasibility. GDQM results are consistent 
TABLE II

Calculated Pull-In Voltages From the GDQM Model as a FUNCTION OF VARIOUS EFFECTS FOR A FIXED-FIXED BEAM WITH $L=250 \mu \mathrm{m}$

\begin{tabular}{|c|c|c|c|}
\hline \multicolumn{2}{|c|}{ Effects } & $V_{\text {Pull-in }}$ & Effectiveness (\%) \\
\hline \multicolumn{2}{|c|}{ Without effects } & 39.1 & N/A \\
\hline \multicolumn{2}{|c|}{ Fringing Field } & 39.03 & 0.2 \\
\hline \multicolumn{2}{|c|}{ Trapezoidal Cross Section } & 38.86 & 0.61 \\
\hline \multirow{2}{*}{ Residual Stress } & $\mathrm{R}=100 \mathrm{MPa}$ & 57.15 & 46.16 \\
\hline & $\mathrm{R}=-25 \mathrm{MPa}$ & 33.13 & 15.27 \\
\hline \multicolumn{2}{|c|}{ Axial Stress } & 39.3 & 0.51 \\
\hline
\end{tabular}

TABLE III

Calculated Pull-In Voltages From the GDQM Model as a FUNCTION OF VARIOUS EFFECTS FOR A FIXED-FIXED BEAM WITH $L=350 \mu \mathrm{m}$

\begin{tabular}{|c|c|c|c|}
\hline \multicolumn{2}{|c|}{ Effects } & $V_{\text {Pull-in }}$ & Effectiveness (\%) \\
\hline \multicolumn{2}{|c|}{ Without effects } & 19.70 & N/A \\
\hline \multicolumn{2}{|c|}{ Fringing Field } & 19.23 & 2.30 \\
\hline \multicolumn{2}{|c|}{ Trapezoidal Cross Section } & 19.54 & 0.81 \\
\hline \multirow{2}{*}{ Residual Stress } & $\sigma_{0}=100 \mathrm{MPa}$ & 35.62 & 44.69 \\
\hline & $\sigma_{0}=-25 \mathrm{MPa}$ & 13.10 & 33.50 \\
\hline \multicolumn{2}{|c|}{ Axial Stress } & 20.3 & 3.05 \\
\hline
\end{tabular}

TABLE IV

COMParison of CALCUlated Pull-IN Voltages For FIXED-FIXED BEAMS WITH $L=250 \mu \mathrm{m}$

\begin{tabular}{|c|c|c|c|}
\hline Stress (MPa) & 0 & 100 & -25 \\
\hline $\begin{array}{c}\text { V proposed } \\
\text { model } \\
(\mathrm{GDQM})\end{array}$ & 39.13 & 57.62 & 33.63 \\
\hline $\begin{array}{c}\mathrm{V}(2 \mathrm{D}) \\
{[28]}\end{array}$ & 39.50 & 56.90 & 33.70 \\
\hline $\begin{array}{c}\mathrm{V}(\mathrm{MEMCAD}) \\
{[28]}\end{array}$ & 40.10 & 57.60 & 33.60 \\
\hline $\begin{array}{c}\Delta(\%) \\
(\mathrm{GDQM} v \mathrm{vs} \\
\text { MEMCAD) }\end{array}$ & 2.40 & 0.03 & 0.09 \\
\hline
\end{tabular}

with the results obtained using MEMCAD [28]. Six fixed-fixed beam models with different residual stresses were simulated and compared in Tables II and III. The geometric and material properties are given as follows [28]: Young's modulus $E$ is 169 GPa, Poisson's ratio is 0.06 , width of the beam $w$ is $50 \mu \mathrm{m}$ for the simple beam, beam thickness $t$ is $3 \mu \mathrm{m}$, initial gap $g$ is $1 \mu \mathrm{m}$, and the permittivity of air is $8.85 \mathrm{pF} / \mathrm{m}$. As shown in Tables II and III, the dominant effect (as measured by the quantity called "effectiveness") is residual stress. "Effectiveness" is defined as the ratio of the pull-in voltage with the effect to the pull-in voltage without the effect and, therefore, gives a quantitative measure as to the importance of each effect on pullin behavior. The results in Tables IV and V indicate that pull-in voltages calculated from the proposed GDQM agree well with the results calculated from the 2-D distributed model and the 3-D MEMCAD model [28]. In this paper, only 21 grid points of GDQM were required to calculate the pull-in voltages within a tolerance of $10^{-12}$ in less than ten iterations. Figs. 5 and 6 show the center gap versus voltage according to Tables II and III and demonstrate that by increasing the residual stress, the pull-in voltage is increased.
TABLE V

Comparison of CALCUlated PUll-IN Voltages For FIXED-FIXED BEAMS WITH $L=350 \mu \mathrm{m}$

\begin{tabular}{|c|c|c|c|}
\hline Stress (MPa) & 0 & 100 & -25 \\
\hline $\begin{array}{c}\text { V proposed } \\
\text { model } \\
(\text { GDQM })\end{array}$ & 20.36 & 35.99 & 13.60 \\
\hline $\begin{array}{c}\text { V(2D) } \\
{[28]}\end{array}$ & 20.20 & 35.40 & 13.80 \\
\hline $\begin{array}{c}\text { V (MEMCAD) } \\
{[28]}\end{array}$ & 20.30 & 35.80 & 13.70 \\
\hline $\begin{array}{c}\Delta(\%) \\
(\text { GDQM vs } \\
\text { MEMCAD) }\end{array}$ & 0.2 & 0.5 & 0.7 \\
\hline
\end{tabular}

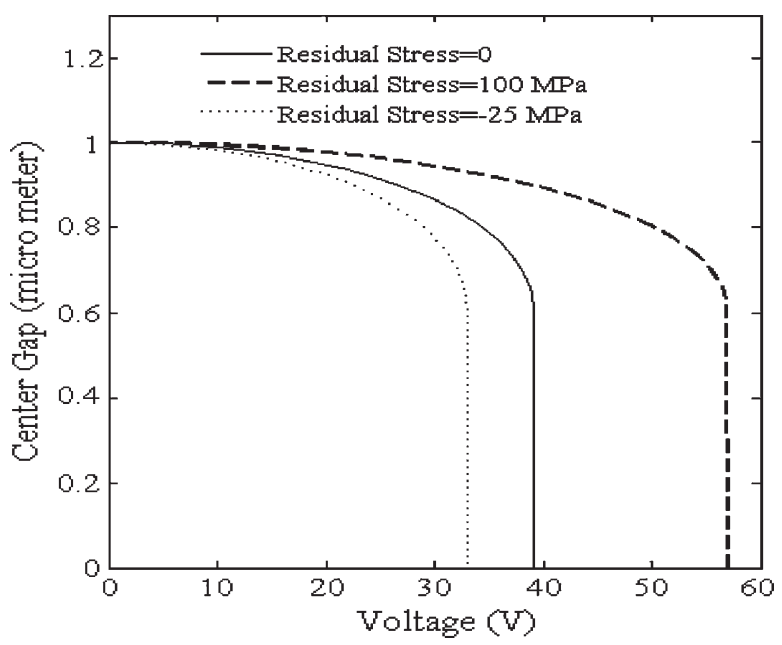

Fig. 5. Center gap versus voltage for fixed-fixed beams with $250-\mu \mathrm{m}$ length and different residual stresses from Table II generated by MATLAB 7 using GDQM

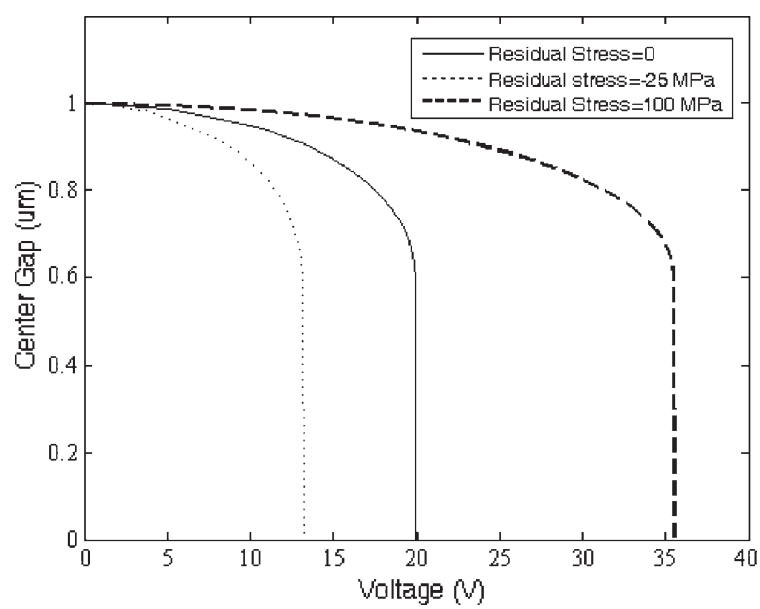

Fig. 6. Center gap versus voltage for fixed-fixed beams with $350-\mu \mathrm{m}$ length and different residual stresses from Table III generated by MATLAB 7 using GDQM.

\section{CONCLUSION}

The design and pull-in simulation of both cantilever and fixed-fixed beam MEMS switches has been implemented using the GDQM and compared with other models as well as with the experimental results. This model captures all important effects that exist on these structures along with their "effectiveness" and shows that the residual stress is the most dominant. 
GDQM was developed and presented, wherein a few grid points and Newton-Raphson iterations are implemented to achieve convergence for various beam types. Pull-in results of both cantilever and fixed-fixed beams reveal that the GDQM algorithm is more efficient than other methods for analyzing pull-in behavior of nonlinear electromechanical coupled systems.

\section{REFERENCES}

[1] S. Senturia, Microsystem Design. Norwell, MA: Kluwer, 2001.

[2] J. I. Seeger and S. B. Crary, "Stabilization of electrostatically actuated mechanical devices," in Proc. Transducers, 1997, pp. 1133-1136.

[3] P. M. Osterberg, H. Yie, X. Cai, J. White, and S. Senturia, "Self-consistent simulation and modelling of electrostatically deformed diaphragms," in Proc. IEEE Conf. Micro Electro Mech. Syst., Osio, Japan, Feb. 25-28, 1994, pp. 28-32.

[4] J. R. Gilbert, R. Legtenberg, and S. D. Senturia, "3D coupled electromechanics for MEMS: Applications of CoSolve-EM," in Proc. MEMS, Amsterdam, The Netherlands, Feb. 1995, pp. 122-127.

[5] R. Legtenberg, J. Gilbert, S. Senturia, and M. Elwenspoek, "Electrostatic curved electrode actuators," J. Microelectromech. Syst., vol. 6, no. 3, pp. 257-265, Sep. 1997.

[6] E. S. Hung and S. D. Senturia, "Leveraged bending for full-gap positioning with electrostatic actuation," in Proc. Solid-State Sens., Actuator Workshop, Hilton Head, SC, 1998, pp. 83-86.

[7] E. S. Hung and S. D. Senturia, "Extending the travel range of analog-tuned electrostatic actuators," J. Microelectromech. Syst., vol. 8, no. 4, pp. 497505, Dec. 1999

[8] H. Sadeghian and G. Rezazadeh, "Some design considerations on the electrostatically actuated fixed-fixed end type MEMS switches," J. Phys.: Conf. Ser., vol. 34, pp. 174-179, 2006.

[9] H. Sadeghian and G. Rezazadeh, "The influence of stress gradient on the pull-in phenomena of microelectromechanical switches," J. Phys.: Conf. Ser., vol. 34, pp. 1117-1122, 2006.

[10] H. Sadeghian, G. Rezazadeh, E. Malekpour, and A. Shafipour, "Pull-in voltage of fixed-fixed end type MEMS switches with variative electrostatic area," Sens. Transducers, vol. 66, no. 4, pp. 526-533, Apr. 2006.

[11] G. Rezazadeh, H. Sadeghian, and E. Abbaspour, "A comprehensive model to study nonlinear behaviour of multilayered micro beam switches," Microsyst. Technol., vol. 14, no. 1, p. 143, 2008. DOI: 10.1007/s00542007-0404-3.

[12] G. Rezazadeh, "A comprehensive model to study nonlinear behaviour of multilayered micro beam switches," Microsyst. Technol., vol. 14, no. 1, pp. 135-141, 2008. DOI: 10.1007/s00542-007-0398-x.

[13] S. Pamidighantam, R. Puers, K. Baert, and H. A. C. Tilmans, "Pull-in voltage analysis of electrostatically actuated beam structures with fixed-fixed and fixed-free end conditions," J. Micromech. Microeng., vol. 12, no. 4, pp. 458-464, Jul. 2002.

[14] Y. Nemirovsky and O. Bochobza-Degani, "A methodology and model for the pull-in parameters of electrostatic actuators," J. Microelectromech. Syst., vol. 10, no. 4, pp. 601-615, Dec. 2001.

[15] L. X. Zhang and Y.-P. Zhao, "Electromechanical model of RF MEMS switches," Microsyst. Technol., vol. 9, no. 6/7, pp. 420-426, Sep. 2003.

[16] R. E. Bellman, B. G. Kashef, and J. Casti, "Differential quadrature: A technique for the rapid solution of nonlinear partial differential equations," J. Comput. Phys., vol. 10, no. 1, pp. 40-52, Aug. 1972.

[17] F. Civan and C. M. Sliepcevich, "Differential quadrature for multidimensional problems," J. Math. Anal. Appl., vol. 101, no. 2, pp. 423-443, Jul. 1984.

[18] C. Shu and B. E. Richards, "Parallel simulation of incompressible viscous flows by generalized differential quadrature," Comput. Syst. Eng., vol. 3 , no. 1-4, pp. 271-281, 1992.

[19] J. Y. Park, G. H. Kim, K. W. Chung, and B. Ju, "Monolithically integrated micromachined RF MEMS capacitive switches," Sens. Actuators A, Phys., vol. 89 , no. 1/2, pp. 88-94, Mar. 2001

[20] E. S. Hung and S. D. Senturia, "Generating efficient dynamical models for microelectromechanical systems from a few finite-element simulation runs," J. Microelectromech. Syst., vol. 8, no. 3, pp. 280-289, Sep. 1999

[21] S. D. Senturia, "Microfabricated structures for the measurement of mechanical properties and adhesion of thin films," in Proc. Transducers, Tokyo, Japan, 1987, pp. 11-16.

[22] J. Qian, C. Liu, D. C. Zhang, and Y. P. Zhao, "Residual stresses in microelectro-mechanical systems," J. Mech. Strength-Special Issue MEMS, vol. 23, no. 4, pp. 393-401, 2001. (in Chinese).
[23] R. K. Gupta, "Electrostatic pull-in test structure design for in-situ mechanical property measurements of microelectromechanical systems (MEMS)," Ph.D. dissertation, MIT, Cambridge, MA, 1997.

[24] T. Mukherjee, G. K. Fedder, and R. D. Blanton, "Hierarchical design and test of integrated microsystems," IEEE Des. Test Comput., vol. 16, no. 4, pp. 18-27, Oct.-Dec. 1999.

[25] W. Chen, C. Shu, W. He, and T. Zhong, "The application of special matrix product to differential quadrature solution of geometrically nonlinear bending of orthotropic rectangular plates," Comput. Struct., vol. 74, no. 1, pp. 65-76, Jan. 2000.

[26] X. Wang and C. W. Bert, "A new approach in applying differential quadrature to static and free vibrational analyses of beams and plates," J. Sound Vib., vol. 162, no. 3, pp. 566-572, Apr. 1993.

[27] Y. C. Hu, C. M. Chang, and S. C. Huang, "Some design considerations on the electrostatically actuated microstructures," Sens. Actuators A, Phys., vol. 112, no. 1, pp. 155-161, Apr. 2004.

[28] P. M. Osterberg and S. Senturia, "M-TEST: A test chip for MEMS material property measurement using electrostatically actuated test structures," J. Microelectromech. Syst., vol. 6, no. 2, pp. 107-118, Jun. 1997.

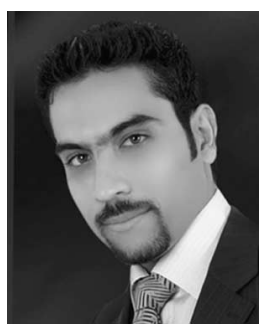

Hamed Sadeghian received the B.S. degree in mechanical engineering from Isfahan University of Technology, Isfahan, Iran, in 2004 and the M.S. degree (with honors) in mechanical engineering from Urmia University, Urmia, Iran, in 2006. He is currently working toward the Ph.D. degree with the Structural Optimization and Computational Mechanics (SOCM) Group, Precision and Microsystems Engineering Department, in collaboration with the Electronic Instrumentation Laboratory, Microelectronics Department, Delft University of Technology,

Delft, The Netherlands.

His current research interests include the instability and dynamic behavior of thin films with ultrafine microstructures, numerical methods, experimental research on MEMS and NEMS, and molecular dynamics and optimization.

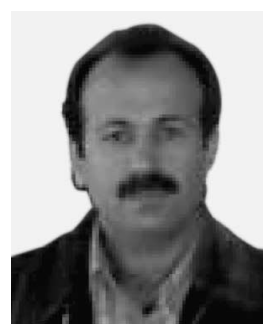

Ghader Rezazadeh was born on March 21, 1965. $\mathrm{He}$ received the B.S. and M.Sc. degrees in mechanical engineering from Isfahan Technical University, Isfahan, Iran, in 1991 and the Ph.D. degree in applied mechanics from the Bauman Moscow Sate Technical University, Moscow, Russia, in 1997.

$\mathrm{He}$ is currently with the Mechanical Engineering Department, Faculty of Engineering, Urmia University, Urmia, Iran, as an Associate Professor. His current research interests include the static and dynamic behavior of MEMS/NEMS structures.

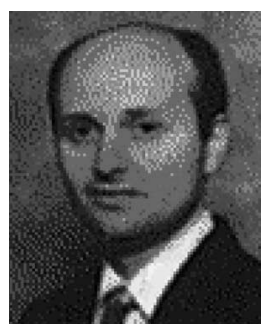

Peter M. Osterberg received the S.B. and S.M. degrees in electrical engineering and computer science in 1980 and the Ph.D. in electrical engineering and computer science in 1995 from the Massachusetts Institute of Technology, Cambridge. His S.B. and S.M. thesis work, which was conducted in the Central Research Laboratory, Texas Instruments, Inc., Dallas, TX, focused on solid-state electromechanical spatial light modulators and deformable mirror displays. His Ph.D. research was in two areas: the simulation and computer-aided design of microelectromechanical systems (MEMCAD) and MEMS material property measurement.

From June 1978 to August 1979, he was with Texas Instruments, Inc. From February 1980 to April 1986, he was with GTE, Inc., Needham, MA, as a VLSI Design/Test Engineering Manager. From April 1986 to August 1991, he was with the Digital Equipment Corporation, Hudson, MA, as a VLSI Product Engineering Manager. He cofounded the consulting firm MaxiMEM Ltd. in 1996. Since 1996, he has been an Associate Professor in the Electrical Engineering Department, University of Portland, Portland, OR. His current research interests include MEMS-based cooling and power generation, and nanoelectronics undergraduate education.

Dr. Osterberg is a member of Tau Beta Pi and Eta Kappa Nu. 